\title{
Energy Saving Control of Solar LED Street Lamp Based on Microcontrollers
}

\author{
Wang Fei \\ Nanchang Institute of Science and Technology, Nanchang, China, 330108
}

Keywords: microcontroller, solar energy, LED street lamp, energy-saving control

\begin{abstract}
In the modern society, the idea of energy saving and emission reduction has gradually permeated people's production and life. In order to achieve this goal, all links in all industries should focus on the implementation of energy saving control. And specifically, the solar LED street lamp based on microcontroller is particularly important. The proposal of the design idea is of great practical significance to the undertakings of environmental protection. In this process, the characteristic analysis on LED light source is a prerequisite. Based on this, an intelligent street lamp driven system based on microcontroller is devised in the paper. Then, it discusses the work process in detail, and finally, works out a controlling methods of LED street lamp suitable to actual circumstances. Operating temperature and external light, for instance, are influencing factors. Only by putting the above requirements into place, can the advantages as well as social value of LED street lamp illuminating system can be fully demonstrated and utilized, injecting a steady stream of driving forces into the environmental protection of China.
\end{abstract}

Compared with traditional lightings, white LED is energy-saving and environment friendly with a relatively long service life. In general, it is a practical and efficient electro-optical light source, which is listed as a major emission reduction measure by the Chinese government. Given the unique applying environment, differences in drive current and operating voltage, the working effect would be different in some degree. In addition, luminous flux will be affected and restricted by various factors in the surrounding contexts, making application of all driving technologies of LED street lamps is less comprehensive. The major role of it is to reduce voltage or resist capacitance in which the parameters of LED will constantly change, leading the adjustment in the end to be difficult. Especially with the increasingly improvement of information technology, living standards have been enhanced accordingly, making the shortcomings of traditional LED driving strategies more prominent. Therefore, it is necessary to develop a set of comprehensive and systematic intelligent drive circuits to create sound operating environment for LED lighting.

\section{Background and significance of the research}

With the strong momentum of energy-saving lighting technology, the total generating capacities in China have exceeded many developed countries, ranking only after the America. Thus, the power consumption in all walks of life is tremendous each year. By the end of 2015, the semiconductor solid-state lights have saved nearly 90 billion kilowatt hours, exceeding the generating capacities of the Three Gorges Dam.

As the socialist market economy develops rapidly in the $21^{\text {st }}$ century in China, the Chinese government has long called for energy conservation and environmental protection, and formulated realistic resource development as well as exploitation schemes so as to promote the harmony, stable development and progress of the society. By the year of 2004, construction departments in both rural areas and national level have drawn up relevant documents in hope of improving the illuminating systems in urban roads. In the process, street lamps will cover 100 percent of roads. For small-medium cities, 95 percent of street lamps will be illuminated, while in large cities, 97 percent of street lamps will be illuminated. The implementation of energy conservation and emission reduction measures in a long time will promote the advance and development of the society. [1] Tt should be noted that, the environmental protection and energy saving are major development directions of future illuminating system, which drives the operation and growth of 
cities, adding momentum to the urban development.

\section{The application and development of microcontroller}

Microcontroller, also called single-chip microcontroller, integrates computer system into the chips rather than using chips with specific logical functions, which is similar to microcomputers.

Just like computers, there are many modules in the microcontroller, for example, parallel bus, CPU and internal storage, but it lacks of I/O equipment. It boasts low price, convenient use, small volume and light weight. Born in the late 1970s, it has undergone three important stages: SoC, SCM, and MCU. Among them, MCU is attached much importance, since it meets the needs of embedded systems at present, realizes the efficient connection between interface circuit and peripheral circuit, highlighting the intelligent control capabilities. [2]

Unlike dedicated processors, microcontroller is more suitable for he embedded system, which is widely promoted. Microcontroller, in fact, has become the largest number of computers worldwide. Today, mechanical products and electron devices all apply microcontrollers such as phone, domestic appliances, palmtops, mobile phones and electronic toys. And an automobile is equipped with more than 40 microcontrollers, so the application of microcontroller has attracted much attention in various fields. [3]

\section{Analysis of system composition}

The paper mainly studies and discusses the design methods of energy-saving solar LED street lamps based on microcontrollers. The solar street lamp is mainly composed of solar panel, controller, pole, LED lamp base and storage batteries. Among them, the controlling system is efficiently controlled by the microcontrollers. The overall design scheme of solar street lamp should take the illuminating needs and climate into consideration as shown in figure 1. [4]

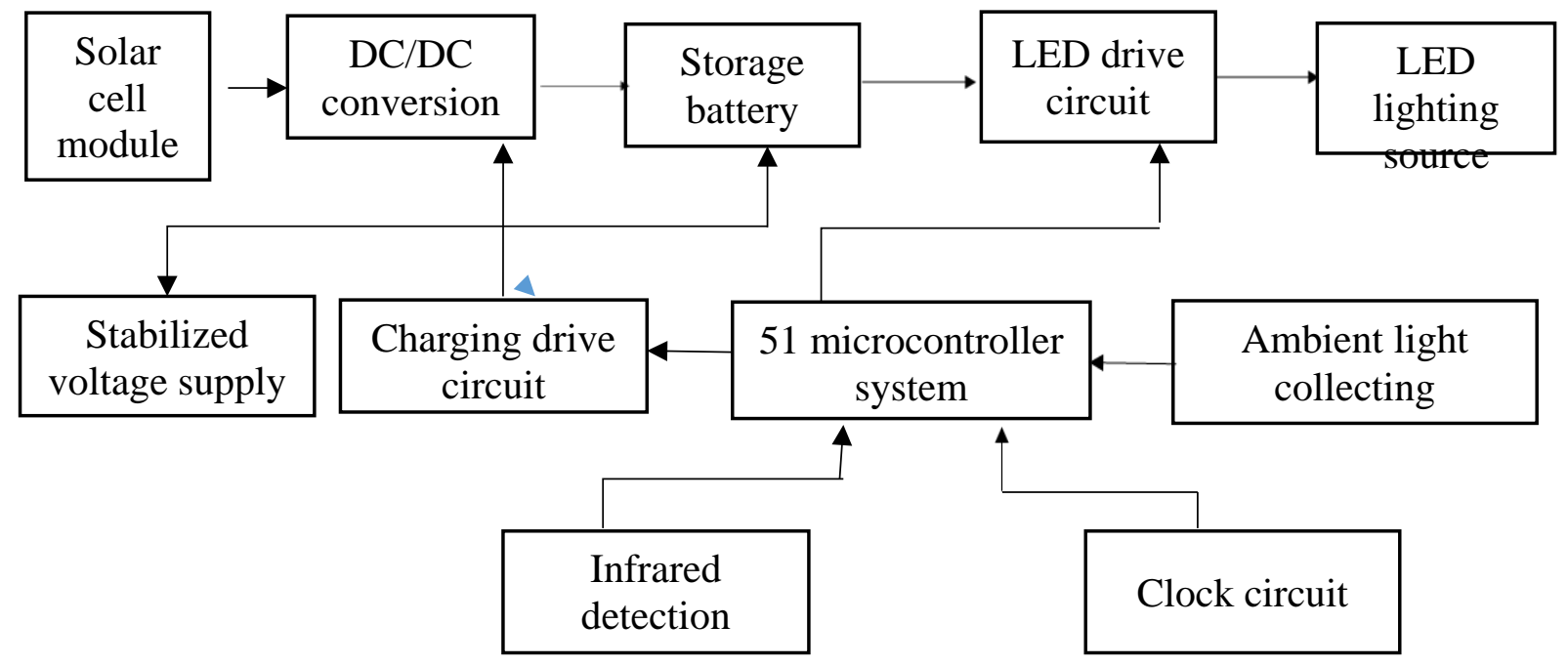

Figure 1 system structure diagram

\subsection{Solar panel}

Solar panel is said to be an important component of solar street lamps with the highest application value, which transforms solar energy in the solar radiation into electric power efficiently, and transmits it into panel to store. There are three common solar panels: amorphous silicon solar cell, single crystalline silicon solar cell and polycrystalline silicon solar cell. And this paper mainly discusses the latter two types. Single crystalline silicon solar cell has stable performance parameters, which is used in rainy south China, while polycrystalline silicon sola cell has simple manufacturing technique with high cost performance, and lower price, which is used in sunny regions with sufficient sunshine. And amorphous silicon solar cell has relatively low requirements for sunshine, which is usually applied in areas without rich sunshine. [5] 
The operating voltage of solar panel is about 1-1.5 times that of the storage battery, ensuring the normal use of the latter. Solar panel has numerous element cells connected in series, whose realistic volume is impacted by total power consumed by line transmission parts and the lighting source. [6] What's more, different places and time will influence the output power of the solar panel. The reference condition refers to the period before and after the noon in sunny days with affluent sunshine so as to ensure the output of solar panels. [7]

\subsection{Solar controller}

No matter the size of the solar lamps, a charge controller with good performance is indispensable. In order to prolong the service life of storage battery, the charging and discharging conditions will be limited to avoid deep charge or overcharge of the storage battery. For example, in regions with great temperature difference, the controller must have the function of temperature compensation, meanwhile, solar controller should have lamp control, temperature control, light control and time control, besides, it can regulate light source to demonstrate its real switching control function so as to prolong the operating time of street lamps in rainy days. [8]

\subsection{The choice of storage battery}

The input energy of solar photovoltaic power system is not stable, therefore, it is often equipped with storage battery system to ensure its operating performance. In most cases, the storage battery will store the power generated by solar panels in rich light efficiently, and release it at night. Then, the author analyzed and summarized the choice of batteries in light of type and volume.

First, the choice of type. One of the common storage batteries is the lead-acid battery, which is applied in most of roads. With low price and relatively low energy, it is sealed, so people do not need to maintain it. However, the prevention against lead-acid pollution should be done. [9] Moreover, maintenance-free lead-acid storage battery, nickel-cadmium battery and average lead-acid storage battery are utilized in small systems, and multidimensional lead-acid storage battery can be applied in practical design.

Second, the choice of volume. If the volume is too small, it cannot meet the real needs of illumination at night; if the volume is too large, the storage battery will be charged for loss, seriously impacting the operating life of battery, even causing waste. Thus, in choosing storage battery, it is necessary to consider the economic and social challenges from a scientific point of view, and pay attention to the prevention against theft of batteries to demonstrate the application value of storage battery in all aspects. [10]

\subsection{Hardware circuit}

The hardware circuit of solar LED street lamps mainly consists of 51 microcontroller system, input and output circuit of switching signal, infrared detection circuit, optoelectronic isolation, clock circuit as well as peripheral circuit.

\subsection{LED intelligent driving system}

The author studied much information and analyzed the operating characteristics of LED driving system. It can be found that, when the current remains unchanged, with the increase of working time, the luminous flux will drop to some extent, and the drive current will directly determine the working voltage. Besides, the difference of temperature will change the number of luminous flux. Generally, the operation of LED luminous system should ensure the stability of temperature as well as the suitability of the output environment. As each LED array will offer different outcomes, it is important to choose the most suitable drive current and working voltage to reach the best working performance. [11-12]

\subsection{Artificial control of 4G system}

In current stage, with the increasingly improving of living standards, and the accelerating of social progress, the performance of $4 \mathrm{G}$ system and intelligent home has been improved. And traditional static system can be updated and innovated to become intelligent tools, and artificial 
control can ensure the intensity and continuity of the LED street lamps. [13-15]

To speak of, intelligent housing system is a subsystem of solar lighting system, however, it can be utilized independently in various conditions to play its crucial role. It can realize the efficient utilization of energy as well as solve many problems, greatly improving the living standards. Particularly in some special conditions, LED lights can be installed to meet lighting needs in different circumstances. Thus, the installment and utilization of intelligent lighting system is notably important in this era. By means of remote control equipment, lighting system can be controlled. While the energy conservation and control of LED street lamps should take this issue into account, gradually achieving the target of environmental protection and energy conservation of LED street lamps.

\section{Conclusions}

To sum up, since the advent of the $21^{\text {st }}$ century, the development of socialist market economy has sped up in China, naturally pushing up the needs for electricity. At the same time, more and more people have realized the importance of utilization of solar energy. This paper, therefore, analyzed and discussed the energy saving methods of LED street lamps. The technology boasts a variety of advantages, for instance, it can transform solar energy into power, which is an important means of future energy utilization. In addition, it is modern, environment friendly, safe without radiation featuring relatively long working life, which is a typical green energy. While the research and development of solar LED street lamps in China is still in the early stage with many defects, the price is much higher than other street lamps. Despite such weakness, it is believed that, a way suitable for solar LED street lamps will be found in the future to inject a stream of vitality into China's environmental protection cause.

\section{References}

[1] Yu Faping, Zhang Xing, Wang Guohua. Solar LED lighting system based on adaptive PI control, PWM constant current controller [J]. Journal of solar energy,2013,27(2):132-135.

[2] Zhao Fuqiang, Chen Fengying, Li Xin, et al. Design of solar LED plant growth lamp [J]. Journal of lighting engineering,2016,27(2):130-132.

[3] Guo Fengyi, He Guangjie, Liu Dan, et al. Design and implementation of photovoltaic street lighting system based on single-chip microcomputer [J]. Computer measurement and control,2013,21(6):1615-1617,1627.

[4] Huang Meng. Design and application of multi-functional solar LED insecticide lamp for greenhouse. [J]. Anhui agricultural science,2014,42(32):11330-11331,11343.

[5] Jia Ruixia, Wang Lijuan. Design of solar LED street lamp controller based on single-chip microcomputer [J]. Electronic test,2013,15(18):11-12.

[6] Kang Lei, Jin Yu, Sang Zhiwei. Automatic scanning LED display based on solar power [J]. Journal of electrical and electronic teaching,2013,35(2):51-53.

[7] Wang Caifeng, Gao Shankun. Solar LED intelligent street lighting system based on single-chip microcomputer [J]. Electronic world, 2011,26(19):77-78.

[8] Yu Li. Design of solar LED lawn lamp controller based on single-chip microcomputer [J]. Technology horizon,2014,11(13):314-314,341.

[9] Wu Xiaorui, Wang Linming. Design and implementation of solar power LED street lamp control system [J]. Electronic production, 2012,12(6):16-17.

[10] Yang Ming. design and implementation of photovoltaic street lighting system based on single chip microcomputer [J]. Heilongjiang science, 2011,8(14):168-169 
[11] Chen Fengran. Design of intelligent solar LED display based on single-chip microcomputer [J]. Science and technology economics guide,2016,10(16):73-73.

[12] Wu Lili, Zhang Yanping. Design of LED solar lamp control circuit based on HT46R23 single chip [J]. China lighting electrical appliance,2016,15(9):25-27,35.

[13] Zhuang Liangqian, Xiao Zhihong, Shen Jun et al. Design of intelligent multi-band LED decoy system based on single-chip microcomputer [J]. Electronic technology,2014,27(6):134-136.

[14] Zhang Hongyu, Jia Sumei, Jiang Shaojun. Design and implementation of solar LED street lamp system based on the Internet of things [J]. Power technology, 2011,41(7):1039-1041,1059.

[15] Cao Weifeng, Duan Xianxing, Hu Zhihong. Design of high-power solar LED street lamp control system [J]. Power technology, 2013, 37(9):1608-1610 1613. 